\title{
sciendo
}

RESEARCH PAPERS FACULTY OF MATERIALS

SCIENCE AND TECHNOLOGY IN TRNAVA

SLOVAK UNIVERSITY OF TECHNOLOGY

IN BRATISLAVA

2019, Volume 27, Number 45

DOI 10.2478/rput-2019-0031

\section{MEASUREMENT OF THE ACOUSTIC ABSORPTION COEFFICIENT BY IMPEDANCE TUBE}

\author{
Eva LABAŠOVÁ ${ }^{1}$, Rastislav ĎURIŠ ${ }^{1}$ \\ ${ }^{1}$ SLOVAK UNIVERSITY OF TECHNOLOGY IN BRATISLAVA, \\ FACULTY OF MATERIALS SCIENCE AND TECHNOLOGY IN TRNAVA, \\ INSTITUTE OF APPLIED INFORMATICS, AUTOMATION AND MECHATRONICS, \\ Ulica JÁnA BotTu 2781/25, 91724 TRNAVA, SLOVAK REPUBLIC \\ e-mail: eva.labasova@stuba.sk, rastislav.duris@stuba.sk \\ Received 23 August 2019, accepted: 10 October 2019, published 29 November 2019
}

\begin{abstract}
The contribution deals with measurement of acoustic absorption coefficient for different single or double-layer materials: cork, two layers of polyethylene, polyethylene and felt. The measurement was performed on an impedance tube of our own construction, using the twomicrophone method transfer function (ISO 10534-2: 1998) and the PULSE measuring system. Values of the acoustic absorption coefficient for the frequencies from $100 \mathrm{~Hz}$ to $1600 \mathrm{~Hz}$ were determined experimentally. Subsequently, those values were processed graphically.
\end{abstract}

Key words

Impedance tube, acoustic absorption coefficient, transfer function, system PULSE

\section{INTRODUCTION}

Nowadays, requirements for reducing noise keep increasing. Equipment manufacturers focus on minimizing noise generation as an important aspect of product design. Testing of the acoustic properties of materials is becoming increasingly important in a wide range of industries.

The acoustic properties of materials such as acoustic absorption coefficient, acoustic reflection coefficient or transient loss (sound reduction) are determined experimentally. Several methods can be used to determine the acoustic properties of materials. Procedure to ensure accuracy and repeatability of measurement of acoustic properties of materials and processing of measured results are prescribed by normative regulations of ISO 10534-1(standing wave ratio method), ISO 10534-2, ASTM E1050-12 (transfer function method for measurement of absorption coefficient, reflectance, acoustic impedance and admittance) and ASTM E2611-09 (transient loss measurement) [1].

Brüel \& Kjær Company has developed a set of impedance tubes type 4206 for the $50 \mathrm{~Hz}$ $6400 \mathrm{~Hz}$ frequency range, including software and hardware solutions, for complex acoustic 
measurement of materials. The impedance tubes are currently a global standard for measuring devices in the field.

However, there is a large number of uniquely designed impedance tubes, especially from the university environment. Based on the study of literature, a prototype impedance tube for measurement of acoustic properties in the frequency range from $100 \mathrm{~Hz}$ to $2 \mathrm{kHz}$ was designed and tested in our workplace [2]. The measuring system is built using the PULSE system of Brüel \& Kjær Company. From the measurements carried out so far, the measured values of the acoustic absorption and reflectance coefficients corresponded to the results obtained by certified measurements.

\section{IMPEDANCE TUBE WITH TWO MICROPHONES}

The impedance tube is the most often used to determine the acoustic absorption coefficient of material samples. There is a sound source at one end of the tube, and a testing sample at the other end of the tube. The absorption measurement is based on the measurement of the interaction between acoustic pressure of the incident sound wave and the reflected sound wave of the plane-wave at the locations of the microphones [3]. There are the two-, three- or fourmicrophone methods according to the number of microphones used in the measurement. Figure 1 shows a conceptual scheme for an impedance tube with two microphones.

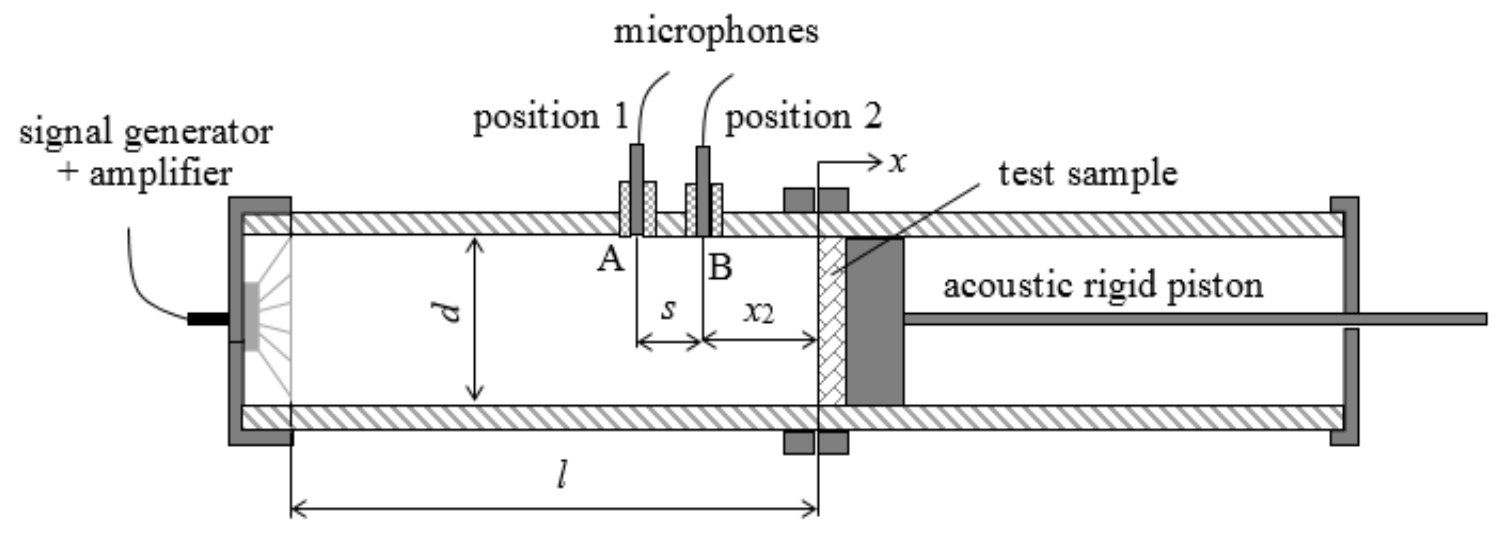

Figure 1 Scheme of impedance tube with two microphones

According to ISO 10534-2 Standard, the impedance tube must be sufficiently long to generate plane sound waves between the source and the sample. The tube must have a uniform cross -section along its entire length and smooth walls without pores and notches. For a tube with a circular cross-section, it is recommended to choose a length greater than 3 times the diameter of the tube [4]. Internal diameter of the tube used in the measurement was $d=0.088$ $\mathrm{m}$, and the tube length (i.e. the distance of the sample from the source) was $l=0.758 \mathrm{~m}$.

The standard recommends using microphones of the same type designed for free sound fields, and diameter less than $20 \%$ of the distance between them for impedance tubes of circular cross-section. Microphones of type 4189-A-031 (1/2 inch microphone for open-field with preamplifier type $2671,20 \mathrm{~Hz}$ to $20 \mathrm{kHz}$, pre-polarized) were used for the tests; their mutual distance was $s=0.065 \mathrm{~m}$. The benefits of using the microphones include: improved stability and accuracy, ease of use and TEDS technology.

The range of the work frequency $f$ is selected in the range $\left(f_{l}, f_{u}\right)$, where $f_{l}$ and $f_{u}$ are the lower and upper frequency level. The lower frequency level is limited by the accuracy of the signal processing equipment. The upper frequency level depends on the inner diameter of the tube $d$ and the sound velocity $c_{0}$. Its value is recommended by the following equation [1]: 


$$
f_{u} d \leq 0.58 c_{0}
$$

Acoustic properties of the selected materials were tested using the constructed impedance tube in the frequency range from $100 \mathrm{~Hz}$ to $1600 \mathrm{~Hz}$.

\section{MEASUREMENT ASSEMBLY AND DETERMINATION OF THE ACOUSTIC ABSORPTION COEFFICIENT}

The wave field in the tube is characterized by the acoustic pressure $p(x)$ of the incident wave $p_{i}(x)$ and the reflected wave $p_{r}(x)$ from the sample:

$$
p(x)=p_{i}(x)+p_{r}(x)=A e^{-j k x}+B e^{j k x},
$$

where $A, B$ are amplitudes of the acoustic pressures of the incident and reflected wave.

The reflection factor $r$ is equal to the ratio of the amplitudes of the sound pressure of the reflected and incident wave $r=B / A$. The reflection factor $r$ is considered as a complex number regarding the possibility of a phase shift of the waves.

The acoustic absorption coefficient $\alpha$ and the reflection factor $r$ (acoustic reflection coefficient) are dimensionless quantities and their values are in the range of $\langle 0,1\rangle$, while it holds [1]:

$$
\alpha=1-|r|^{2}
$$

In case that the material absorbs all incident energy, the acoustic absorption coefficient $\alpha=1(r=0)$. Materials of porous or fibrous structure are recommended for absorption of sound. In case that the material perfectly reflects all incident sound waves from the surface, the acoustic absorption coefficient $\alpha=0(r=1)$.

For the calculation of the reflection factor:

$$
r=r_{r}+j r_{i}=\frac{H_{12}-e^{-j k s}}{e^{j k s}-H_{12}} e^{2 j k\left(s+x_{2}\right)},
$$

where:

$j \quad-\quad$ is an imaginary component,

$s \quad$ - is the distance between microphones,

$x_{2} \quad$ - is the distance between the sample and the microphone located in position 2,

$k \quad$ - is the wave number,

$H_{12}$ - is the transfer function between positions 1 and 2.

In general, for a complex acoustic transmission function, the following equation holds:

$$
H_{12}=\frac{p\left(x_{2}\right)}{p\left(x_{1}\right)}=\frac{S_{12}}{S_{11}}=H_{r}+j H_{i}
$$

where:

$H_{r} \quad$ - is the real component of the transfer function $H_{12}$,

$H_{i} \quad$ - is an imaginary component of the transfer function $H_{12}$,

$S_{11} \quad$ - is the power auto-spectral density of the signal (from microphone A),

$S_{12}$ - is the cross power spectral density of the signal (from microphones A and B).

In case that white noise is used to activate acoustic waves in the tube, ASTM E1050-12 Standard recommends the following equation to determine the transfer function:

$$
H_{12}=\frac{S_{22}}{S_{21}}=H_{r}+j H_{i}
$$


where:

$S_{22}$ - is the power auto-spectral density of the signal (from microphone B),

$S_{21} \quad$ - is the cross power spectral density of the signal (from microphones B and A).

Figure 2 shows a diagram of a measurement apparatus used to determine the acoustic absorption coefficient. The measurement apparatus consists of the following components:

- display unit - computer,

- hardware unit of Brüel \& Kjær FFT Analyze type 3560-B-120 (frontend),

- software unit of Brüel \& Kjær PULSETM Labshop,

- Kundt impedance tube,

- two measuring microphones of Brüel \& Kjær type 4189-A-021,

- amplifier of Brüel \& Kjær type 2706.
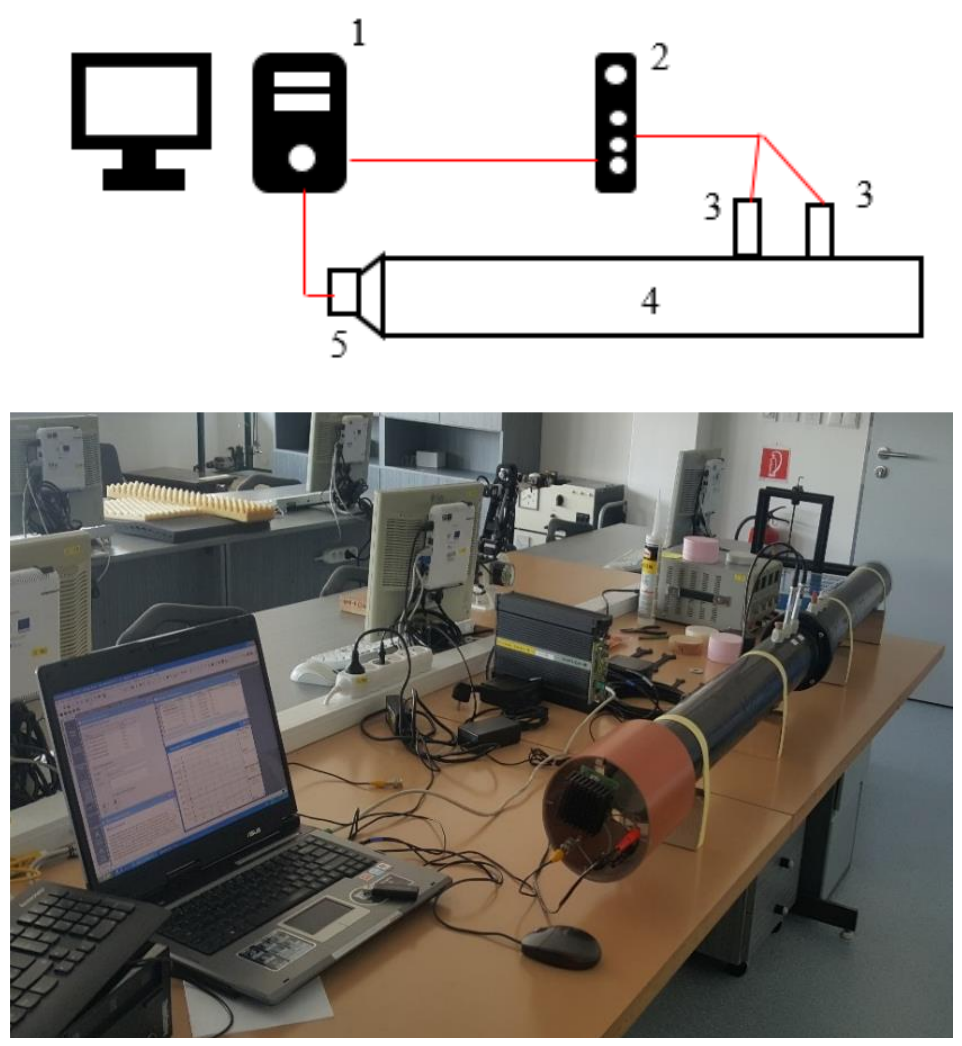

Figure 2 Measurement apparatus and its diagram

$1-P C$, sound card, display unit, 2 - hardware unit PULSE, 3 - microphones,

4 - impedance tube, 5 - amplifier

\section{DETERMINATION OF TRANSFER FUNCTION AND PROCEDURE OF MEASURING}

To determine the transfer function, it is necessary to measure the acoustic pressure in the microphones under different conditions. First, the measurement is performed without white noise. In the next step, the measurement is performed with a white noise generator connected to the tube's speaker. This measurement is performed twice. For the first time, the microphones A, B are in positions 1, 2 as shown in Figure 1 (Figure 3, curve $a$ ). For the second time, the measurement is performed while changing positions of microphones. Microphone $\mathrm{A}$ is in position 2 and microphone $\mathrm{B}$ is in position 1 (Figure 3, curve $b$ ). The measured data is processed by the Pulse LabShop software; the transfer function $\mathrm{H}_{12}$ is the result (Figure 3). If measurements are performed in the right way, we get a transfer function characterized by symmetry around the horizontal axis. 
The microphones are then returned back to the starting positions. Subsequently, test sample can be placed inside the tube and measurement can be made while transmitting white noise into the tube. The result is a graphical representation of the absorption coefficient for the sample in the set-up software project. From the graph, we can read the coefficient values at individual frequencies, which can be subsequently processed in a tabular form. Figure 4 shows the variation of the acoustic absorption coefficient for one of the test samples.

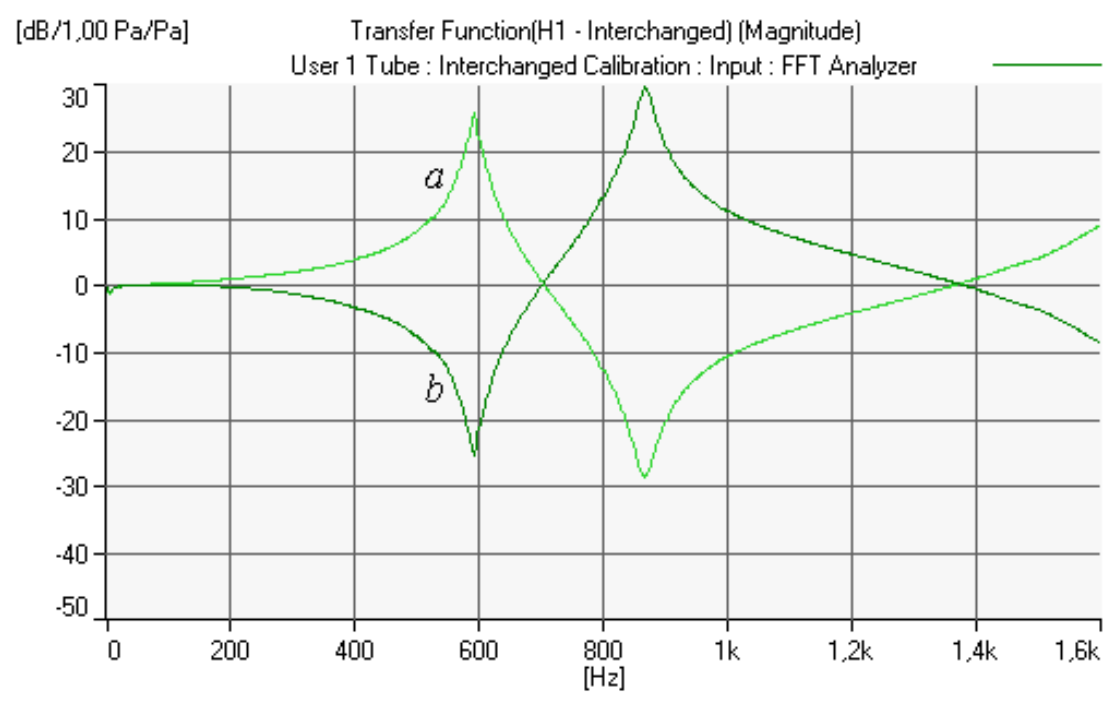

Figure 3 Transfer function

Absorption Coefficient (AvglMeasurement, User 1 - A)

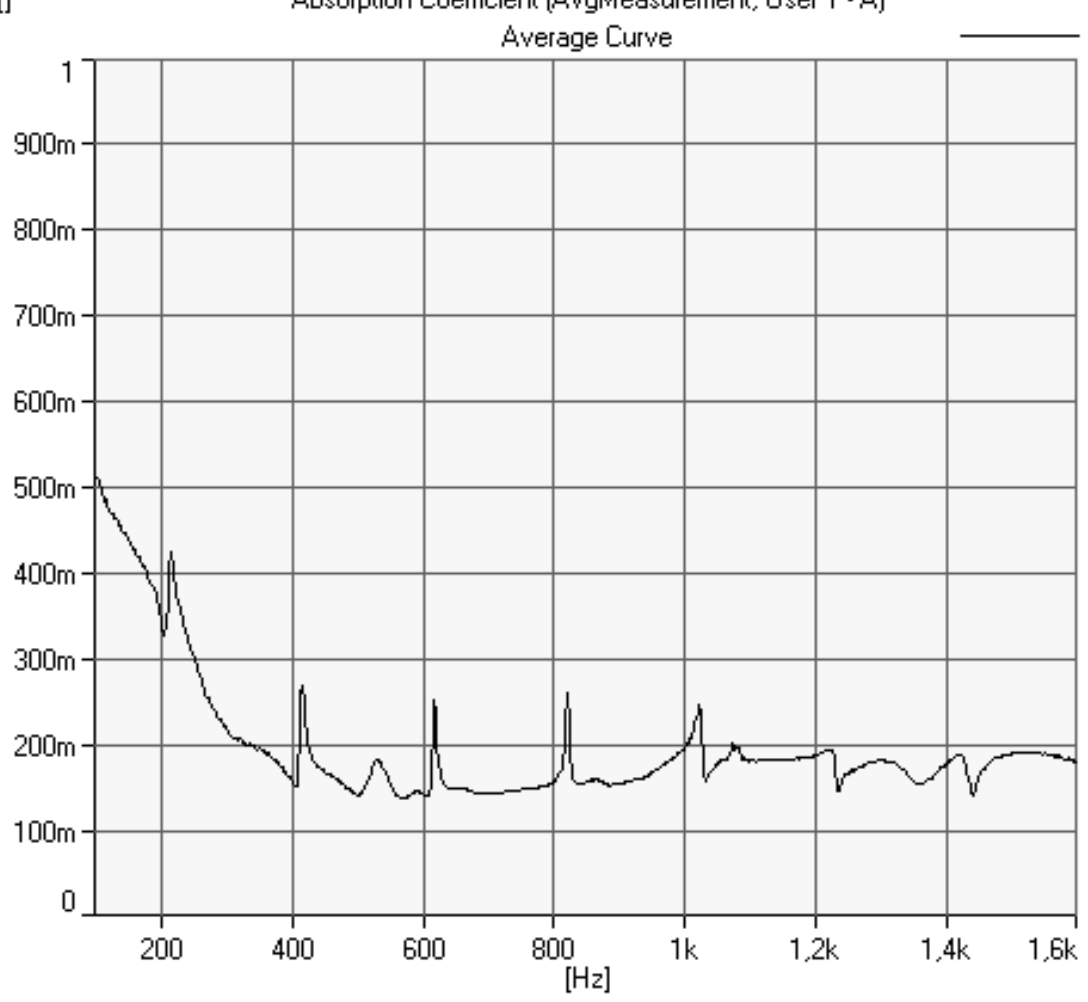

Figure 4 Graphical variation of acoustic absorption coefficient $\alpha$ (sample: cork, thickness $26.5 \mathrm{~mm}$ )

\section{ANALYSIS OF ATTAINED RESULTS}

The values of absorption coefficient of selected samples are presented in this paper. All samples were of the same diameter, which corresponded to the inner diameter of the tube 
$d=0.088 \mathrm{~m}$. The thickness of the samples was different. The density of the samples was determined by the mass and dimensions of samples. Data of samples is shown in Table 1 . The way the layers were arranged in the samples is shown in Table 1, too.

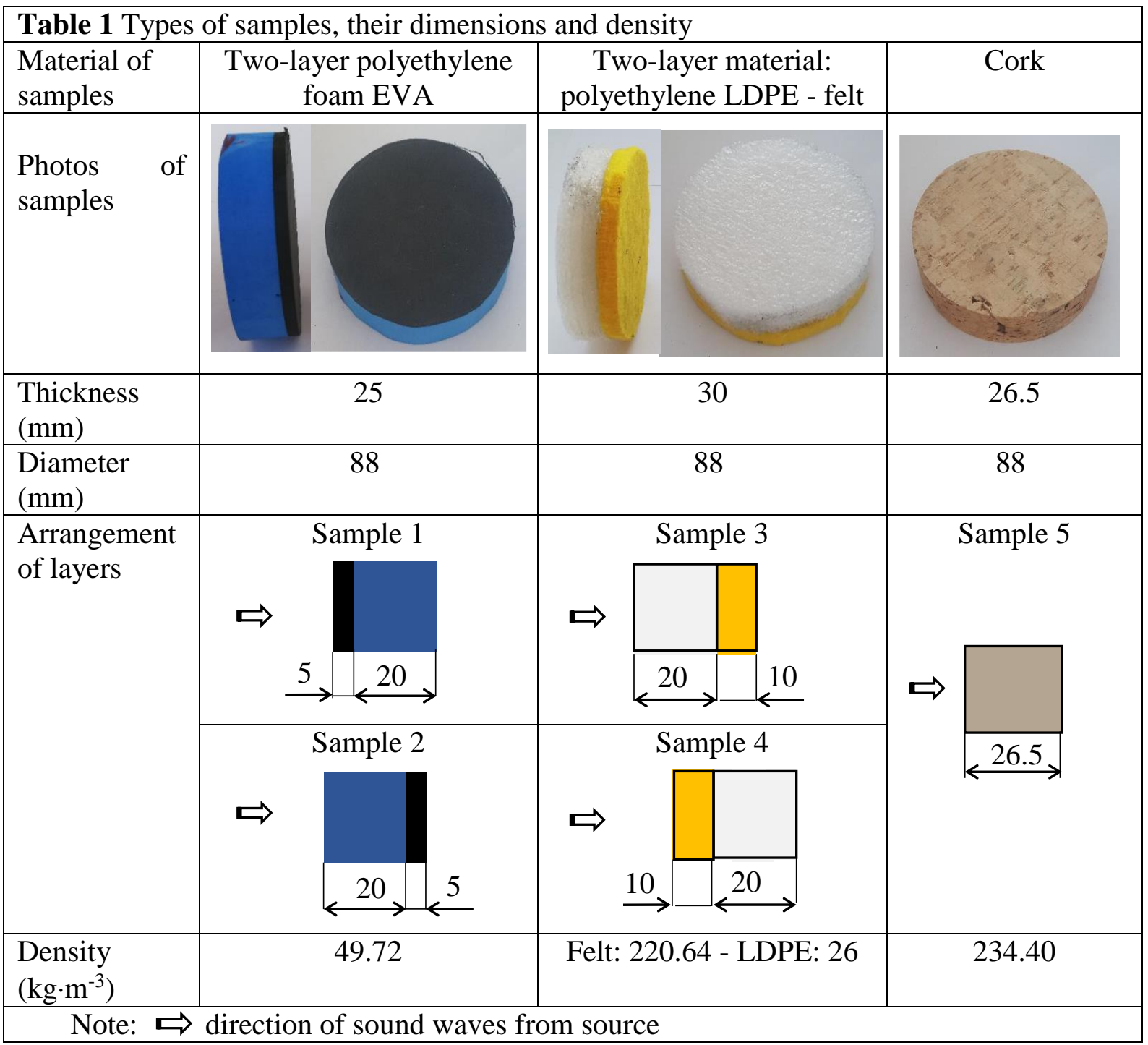

Six measurements were performed for each sample, from which the average value can be determined by the software. The absorption coefficient values were read from the graphs at each frequency for individual sample. The data was put into table and then processed into graph. For better clarity, a graphical record of the values of the acoustic absorption coefficients is shown in this paper, Figure 5.

The materials such as cork or two-layer polyethylene foam absorb sound at lower frequencies, mostly from $100 \mathrm{~Hz}$ to $300 \mathrm{~Hz}$. Two-layer polyethylene foam shows the lowest value of coefficient $\alpha$ at frequencies from 800 to $900 \mathrm{~Hz}$. For this material, there are no large differences in the values of coefficient $\alpha$, depending on the side closer to the noise source. The sample made of cork attained the smallest value of coefficient $\alpha$ at the frequencies of $500 \mathrm{~Hz}$ and $600 \mathrm{~Hz}$.

For a two-layer material (polyethylene - felt), the lowest values of coefficient $\alpha$ is at frequency of $400 \mathrm{~Hz}$. This material attains the highest value of coefficient $\alpha$ at frequencies 700 $\mathrm{Hz}$ and $800 \mathrm{~Hz}$. In general, it may be said that the two layer material polyethylene - felt in which the felt side is closer to the source of noise reaches higher values of acoustic absorption 
coefficient over the whole frequency range compared to the sample in which the polyethylene side is closer to the noise.

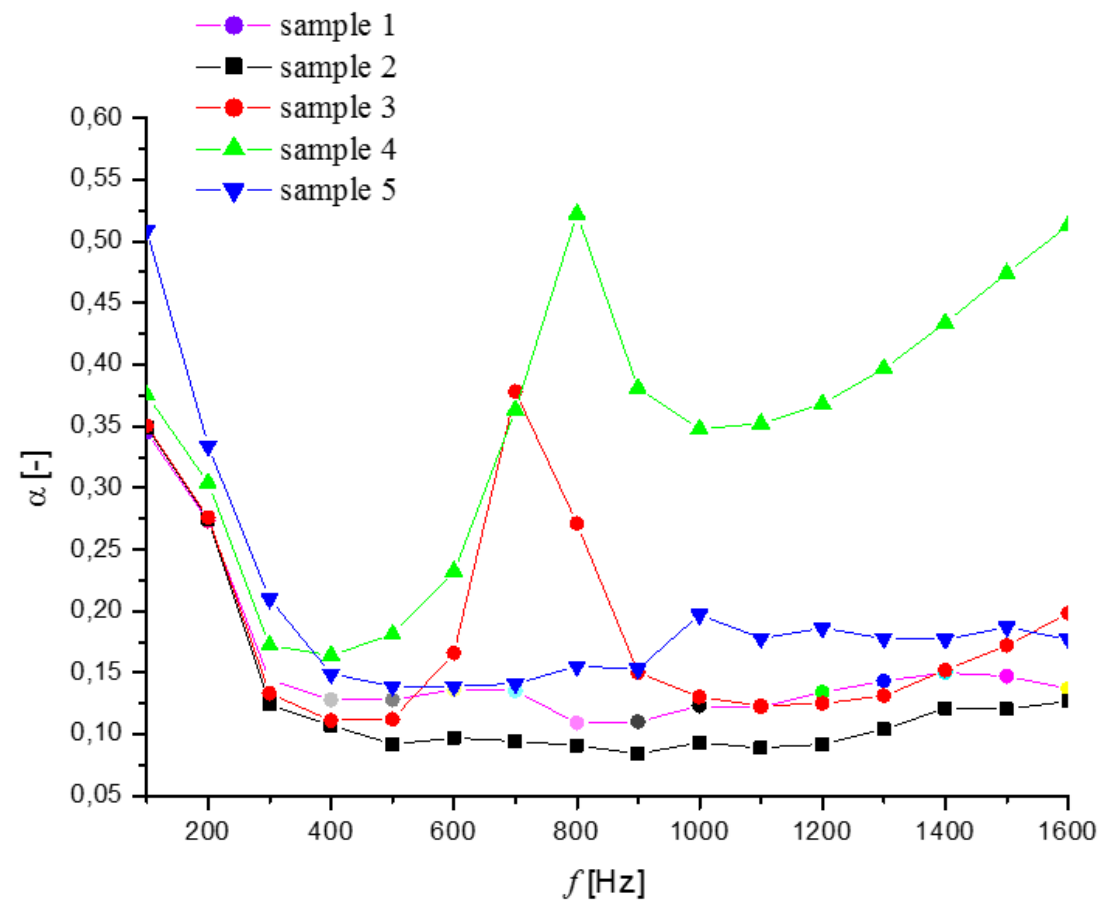

Figure 5 Variations of sound absorption coefficient in dependence on frequency

\section{CONCLUSION}

The acoustic absorption coefficient is a dimensionless quantity and its value is in the range $\langle 0,1\rangle$. If its value is closer to the number one, the absorption properties of the material are better. The selected samples of material show little absorption properties. The value of the coefficient $\alpha$ can probably be increased by increasing the thickness of material. The sample made of cork was of a greater thickness than the samples of the two-layer material, and, over the entire frequency range, its value of coefficient was higher than that of the mentioned samples.

\section{Acknowledgement}

This research output was supported by the KEGA Scientific Grant Agency of the Slovak Republic under the Grant No. 029STU-4/2018.

This publication has been written thanks to support of the Operational Program Research and Innovation for the project: Research, modeling and simulation of industrial production processes using progressive technologies, ITMS code: NFP313010T589 co-financed by the European Regional Development Fund.

This publication has been written thanks to support of the Operational Program Research and Innovation for the project: Research of advanced methods of intelligent information processing, ITMS code: NFP313010T570 co-financed by the European Regional Development Fund. 


\section{References}

[1] ISO 10534-2, 1998, Acoustic - Determination of sound absorption coefficient and impedance in impedance tubes - Part 2: Transfer-function method.

[2] http://www.scs-controlsys.com/systems/pdf/SCS-MT-PRO-KIT-ABSORPTION.pdf, G. Amadasi, Kundt Tube Acoustic Absorption and Transmission Loss [Online]. [Accessed: 012019]

[3] BOROŠOVÁ, L., BADIDA, M., BARTKO, L. 2014. Impedance tube as a tool for measuring and evaluation acoustical noise descriptors. Transfer inovácií (Innovation transfer), 30. ISSN 13377094

[4] http://www.angelofarina.it/Public/Standing-Wave/Absorption_Meas.pdf, Andrew F. Seybert, Notes on Absorption and Impedance Measurements [Online]. [Accessed: 12-2018]

\section{ORCID}

Eva Labašová 0000-0001-9055-1233

Rastislav Duriš 0000-0002-5984-8730 